\title{
Genetically modified artificial antigen-presenting cells (aAPC) for expansion of melanoma tumor infiltrating lymphocytes with optimal properties for adoptive cell therapy
}

\author{
Marie-Andrée Forget ${ }^{1,3^{*}}$, Shruti Malu ${ }^{1}$, Hiu Liu' ${ }^{2}$, Christopher Toth ${ }^{1}$, Sourindra Maiti ${ }^{3}$, Charuta Kale ${ }^{1}$, \\ Chantale Bernatchez ${ }^{1}$, Helen Huls ${ }^{3}$, Ena Wang ${ }^{2}$, Patrick Hwu' ${ }^{1}$, Laurence J Cooper ${ }^{3}$, Laszlo G Radvanyi ${ }^{1}$
}

From Society for Immunotherapy of Cancer 28th Annual Meeting

National Harbor, MD, USA. 8-10 November 2013

Adoptive cell therapy (ACT) with expanded autologous tumor infiltrating lymphocytes (TIL) has emerged to be a powerful salvage therapy for metastatic melanoma with response rates up to $50 \%$. However, the generation of the TIL ACT product is technically challenging with current methods requiring a large excess of allogeneic normal donor (pool) or patient-derived PBMC (200:1 ratio to the TIL) used as "feeders" to support a rapid expansion protocol (REP) to generate the final TIL infusion product. Because PBMC feeder products consist of a heterogenous cell population, it introduces undesired variability in TIL expansion rates and phenotype, especially in the yield of $\mathrm{CD} 8+\mathrm{T}$ cells that are the most active component of the TIL product. Here, we have developed an alternative to PBMC feeders using a K562-based artificial antigenpresenting cell (aAPC) system expressing CD64, CD86, and 4-1BBL that is much more practical and costeffective. Using PBMC feeders as controls, we found an optimal aAPC:TIL ratio (50:1) supporting maximal TIL expansion used for subsequent experiments. Analysis of the resulting TIL products from multiple experiments revealed that the aAPC induced higher rates of CD8+ $\mathrm{T}$ cell expansion with a comparable effector-memory phenotype as TIL expanded with the traditional PBMC feeder approach. The exceptions were CD56 that was more highly expressed on the CD8+ cells and CD28 which had a lower expression. Notably, TIL expanded with aAPC were enriched in CD8 + BTLA + T cells, a

${ }^{1}$ Melanoma Medical Oncology, MD Anderson Cancer Center, Houston, TX, USA

Full list of author information is available at the end of the article subset highly correlated with clinical response to ACT. TCR V $\alpha \beta$ clonotype analysis also found that TIL expansion with the aAPC did not alter the diversity of the $\mathrm{T}$ cell repertoire. Further analysis using gene chip profiling revealed significant differences in gene expression in TIL products expanded using the aAPC versus with PBMC feeders. These included an up-regulation of certain genes in the Wnt pathway, cyclic nucleotide metabolism, and multiple genes in different stem cell pathways. This more stem-like profile may have beneficial properties following adoptive transfer. Finally, we found that CD8+ TIL expanded with aAPC had a similar anti-tumor effector cell activity in CTL and IFN- $\gamma$ release assays. Overall, our data demonstrates that this aAPC system is a suitable alternative to generate clinical-grade melanoma TIL infusion products for ACT that produces comparable and perhaps even more active $\mathrm{T}$ cells than current methods. Our group is currently phasing in the use of these aAPC for our GMP TIL production.

\section{Authors' details}

${ }^{1}$ Melanoma Medical Oncology, MD Anderson Cancer Center, Houston, TX, USA. ${ }^{2}$ Transfusion Medicine, National Institutes of Health, Bethesda, MD, USA. ${ }^{3}$ Pediatrics Research, MD Anderson Cancer Center, Houston, TX, USA.

Published: 7 November 2013

doi:10.1186/2051-1426-1-S1-P8

Cite this article as: Forget et al: Genetically modified artificial antigenpresenting cells (aAPC) for expansion of melanoma tumor infiltrating lymphocytes with optimal properties for adoptive cell therapy. Journal for ImmunoTherapy of Cancer 2013 1(Suppl 1):P8. 